\title{
Erratum to: Hydroxyapatite/regenerated silk fibroin scaffold-enhanced osteoinductivity and osteoconductivity of bone marrow-derived mesenchymal stromal cells
}

\author{
Jia Jiang $\cdot$ Wei Hao $\cdot$ Yuzhuo Li $\cdot$ Jinrong Yao \\ Zhengzhong Shao $\cdot$ Hong $\mathbf{L i} \cdot$ Jianjun Yang $\cdot$ \\ Shiyi Chen
}

Published online: 7 June 2013

(C) Springer Science+Business Media Dordrecht 2013

\section{Erratum to: Biotechnol Lett \\ DOI 10.1007/s10529-012-1121-2}

Subsequent to publication of this paper online the authors have realized that there was an error in Fig. 1f. The authors inadvertently misused SEM images
BMSCs seeded onto a HA/RSF scaffold after 14 days in Fig. 1f. The correct figures are given below. The error in the displayed image does not alter the validity of the work and the conclusions. The authors apologize to readers for these errors.
The online version of the original article can be found under doi:10.1007/s10529-012-1121-2.

J. Jiang $\cdot$ Y. Li $\cdot$ H. Li $\cdot$ J. Yang $\cdot$ S. Chen $(\varangle)$

Fudan University Sports Medicine Center, Department of Sports Medicine and Arthroscopy Surgery, Huashan Hospital, Fudan University, No. 12 Wulumuqi Zhong Road, Shanghai 200040, China

e-mail: chenshiyi888@163.com

W. Hao $\cdot$ J. Yao $\cdot$ Z. Shao

Laboratory of Advanced Materials, National Key

Laboratory of Molecular Engineering of Polymers,

Department of Macromolecular Science,

Fudan University, Shanghai 200433, China 

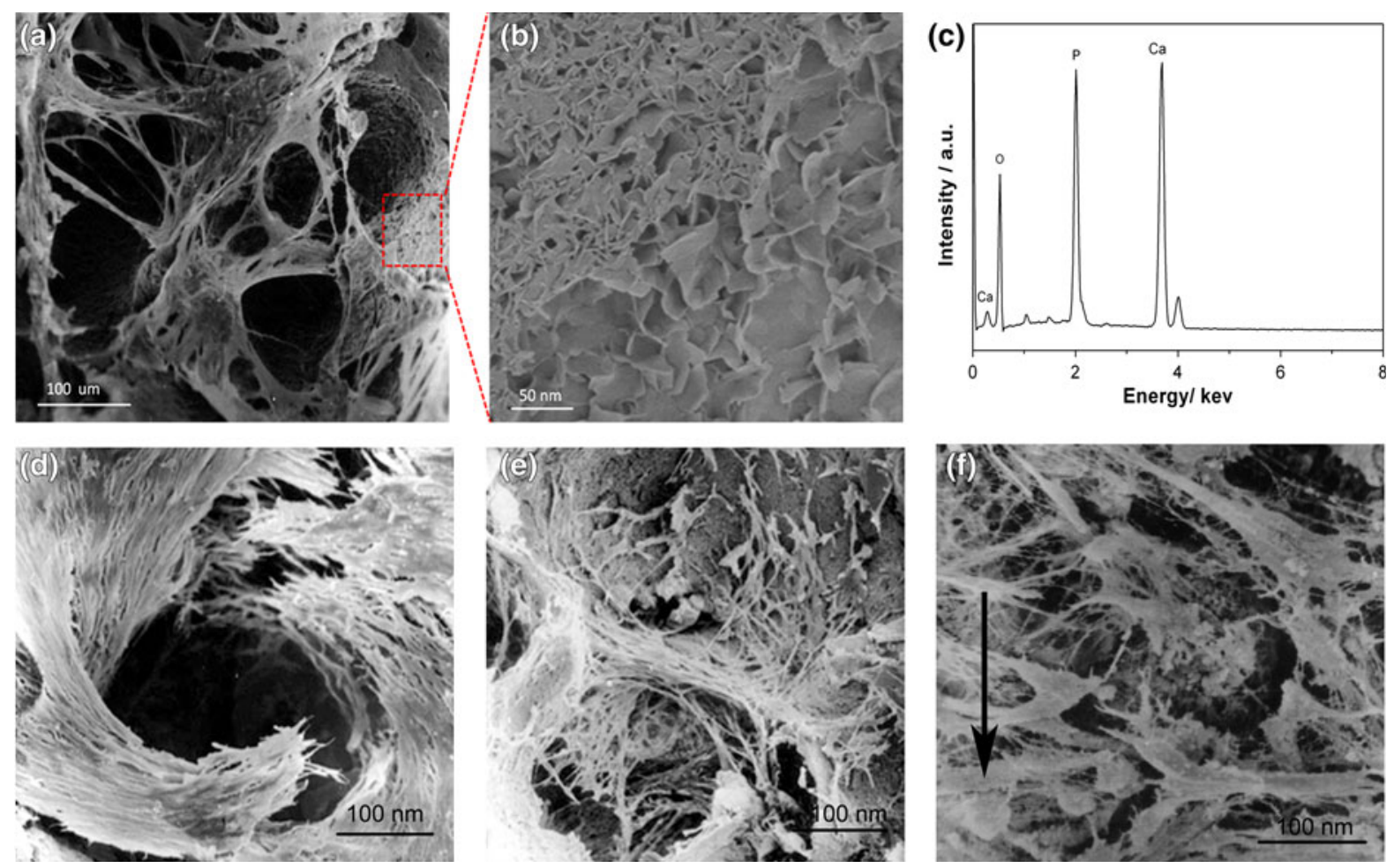

Fig. 1 (a) SEM image and (b) high-magnification SEM image of seeded onto a HA/RSF scaffold after (c) 1 day, (e) 7 days, and a prepared HA/RSF scaffold. (d) Energy dispersive X-ray (EDX) analysis of a prepared HA/RSF scaffold. SEM images of BMSCs (f) 14 days. The blank arrow in (f) indicates typical osteoblastlike cells 